\title{
ON COMPUTING THE BEHAVIOR OF THE MIND FROM AN EASTERN PHILOSOPHICAL PERSPECTIVE
}

\author{
A.S. KARUNANANDA ${ }^{1}$, P.R. GOLDIN ${ }^{2}$, G. RZEVSKI ${ }^{3}$, S. FERNANDO ${ }^{4}$ \& H.R. FERNANDO 4 \\ ${ }^{1}$ General Sir John Kotelawala Defence University, Sri Lanka. \\ ${ }^{2}$ University of California, Davis, USA. \\ ${ }^{3}$ Centre for Complexity Science Applications, The Open University, UK. \\ ${ }^{4}$ Department of Computational Mathematics, University of Moratuwa, Sri Lanka.
}

\begin{abstract}
The human mind is one of the most intriguing complex systems found in nature. Research has been conducted to develop a computer model of the mind from the Theravada Buddhist description detailed in the Abhidhamma literature. Within this context, the mind is identified as a conditional flow of thoughts that is characterized by 52 mental factors, such as perception, volition, attention, anger, attachments, mindfulness and so on. Thus, mental factors are the building blocks of thoughts. Combinations of these 52 mental factors can form 89 different thoughts with characteristics such as feelings, roots, functions, doors, objects and bases. We have developed a computational ontology of Buddhist theory of mind (OntoBM) that describes the complex association among thoughts and mental factors. OntoBM can be primarily used to examine combinations of mental factors that can form thoughts, associations among mental factors and details of thoughts. More importantly, researchers and scholars can also use OntoBM to experiment with the effect of modulation of mental factors on the formation of various thoughts.

Keywords: artificial intelligence, Buddhism, complex systems, simulations.
\end{abstract}

\section{INTRODUCTION}

Nature is full of complex systems [1]. The brain and mind are excellent examples of complex systems. Although brain and mind are distinct terms, they have been used as synonyms in a wider spectrum of subject areas. Numerous brain simulators have been developed to carry out experiments that cannot be done otherwise with actual tissues and cells in a brain [2]. New research directions such as Mind uploading [3] and Blue Brain [4] are excellent attempts to model the brain. Numerous software programs [5] are also available to experiment with brain simulations. On the other hand, advancements in computing, neuroscience and psychology enable study of brain behavior through various technologies such as functional magnetic resonance imaging (fMRI) [6], electroencephalography (EEG) wave signals [7] and transcranial direct current stimulation (tDCS) [8]. Such studies have made a tremendous impact on understanding the structure and function of the human brain. However, limited research has been conducted on computer modeling and simulation of mind $[9,10]$. In particular, very few studies have attempted to use [11,12] computer modeling of mind from the Buddhist philosophical perspectives. We have conducted research to use Abhidhamma [13] in Theravada Buddhist theory of mind for the development of a computational ontology of mind. This paper presents the design and implementation of computational Ontology of Buddhist theory of Mind (OntoBM), which becomes the basis for developing a smart simulator of mind.

The rest of the paper is organized as follows. Section 2 reviews complexity science and computer modeling of complex systems. Section 3 discusses the Buddhist theory of mind. Section 4 presents the design of a computational ontology of mind. Section 5 presents how the ontology of mind can be used in practice. Section 6 concludes the paper with a note on further work. 


\section{WHAT ARE COMPLEX SYSTEMS?}

Complexity science emerged as an attempt to explain and model what is called complex systems. This field has emerged from the pioneering work by Prigogine [14,15]. Complex systems have been defined as the systems with large number of distributed and interconnected entities that manifest under lots of uncertainty in a dynamically changing environment [1]. The brain is a commonly cited example for a complex system, which comprises of 100 billion neurons [16] or processes that are massively interconnected, distributed and are constantly firing under dynamically changing chemical and physical reactions. The Internet, especially the Internet of Things, is also an excellent example for a complex system. As estimated, by 2015, there will be 25 billion devices connected to the Internet of Things and billions of transactions move on the network of networks [17]. Apart from the brain, many other systems including industrial automation, aircraft maintenance and military logistic management are well-known examples for complex systems.

\subsection{Computer modeling of complex systems}

This section discusses suitable computational technologies for modeling complex systems. In this regard, we emphasize the importance of ontology and multi-agent technology.

\subsubsection{Ontology}

Traditionally ontology is the philosophical study of themes, such as being, existence and categories of being [18]. The computing community has borrowed the term ontology from philosophy. There are many definitions about ontology; yet in operational terms, ontology can be seen as a specific conceptualization of domain knowledge [18]. There are ontological solutions for various domains including medicine [19] and automotive industry [20]. There are also numerous tools available for ontology development, mapping, editing, merging and so forth. Protégé (generic framework for ontology development) [21], PROMPT (ontology mapping environment) [22] and automated solution for matching of two ontologies [23] are examples of such tools. It should be noted that ontology is a huge reservoir of knowledge or knowledge base. Thus, reasoning on ontology of complex domains gives rise to another challenge. There are also many tools (e.g. Chainsaw [24], FaCT++ [25] and Pellet [26]) for reasoning on ontology.

\subsubsection{Multi-agent systems}

Multi-agent systems [27,28] technology can be seen as the best computational approach to model complex systems. In this context, all the entities in a complex system can be defined as small programs called agents. Each agent accomplishes its own goal, preference, knowledge and strategies to achieve a certain goal. Numerous examples can be given to show the power of multi-agent systems to model complex systems [1]. For instance, Hongqiao et al. [29] have used multi-agent systems for modeling complex hospital environments. Belgacem and Fates have presented a multi-agent solution for complex termite behavior [30]. Rzevski [28] has shown the application of multi-agent systems technology to model a variety of real-world problems including large-scale societies and huge technological and engineering systems. Numerous toolkits are available for the design and development of multi-agent systems. Probably, Java Agent Development Framework (JADE) [31] is the most popular among them.

\section{BUDDHIST THEORY OF MIND}

Buddhism, more specifically, Buddhist philosophy is primarily aimed at the development of mind in order to achieve freedom from suffering. The teaching of Buddhism comprises three baskets or 
sources of knowledge known as Suttapitaka, Vinayapitaka and Abhidhammapitaka [32,33]. Abhidhamma is the most philosophical, analytical and structured presentation of the doctrine of Buddhism [32]. Abhidhamma is also regarded as Buddhist psychology [32,34]. In literature, Abhidhamma comes under both Theravada and Mahayana traditions of Buddhism. Our research is based on the Theravada tradition of Abhidhamma which is rooted in the ancient Buddhist text known as Abhidammaththa Sangah [33]. Abhidhamma expounds a comprehensive description of 'mind' by showing definition, constitution, roles, mechanism, control and development of mind to achieve various cognitive skills.

\subsection{Mind as a flow of thoughts}

According to Abhidhamma, mind (citta in Pali) is a flow of thoughts. These thoughts are formed on the basis of various perceptions made through sight, sound, smell, touch, taste, thinking and recalling. Thus, in a broader sense, thoughts are of two types, namely, thoughts based on five senses and thoughts based on mind [13]. Each thought is conditioned by previous thoughts. Mind does not have a moment without a thought. To explain this phenomenon more specifically, Buddhist psychology has also identified a special type of thought, namely, life continuum (bhavangacitta) [13], which arises whenever a person does not manifest thoughts through five senses or the mind. For instance, when an individual falls into a dreamless sleep or becomes unconscious, the bhavangacitta manifests. The bhavangacitta is similar to a vehicle resting in a neutral gear when the engine is running. The Buddhist theory of mind suggests that the flow of thoughts in the mind occurs in a sequence of blocks of thoughts, which are known as cittavithi or thought processes. Each thought process begins and ends with bhavangacitta. The concept of thought process elegantly describes the mechanism of the mind. It is beyond the scope of this paper to discuss thought process in detail. A reader interested in thought process may refer to computer modeling of thought process by Karunananda [12].

\subsection{Mental factors}

Abhidhamma describes mental factors that in combination constitute different types of thoughts that arise in the mind [33]. There are 52 mental factors that determine the nature of thoughts. There are altogether 89 (or 121 according to a different classification) possible combinations of these 52 mental factors. As shown in Table 1, 52 mental factors are subdivided under three main categories: 13 ethically variables, 14 unwholesome and 25 wholesome. English translations for 52 mental factors vary. Thus, we have predominantly used the English translations given by Bodhi [33] in our research work.

As shown in Table 1, within 'ethically variables', there are seven universal mental factors that appear in all thoughts. These mental factors include contact (passa), feeling (vedana), perception (sanna), volition (cetana), one-pointedness (ekaggatha), psychic life (jivithedriya) and attention (manasikara). In contrast, 'occasional mental factors' appear in both wholesome and unwholesome thoughts. They are identified as initial application (vithakka), sustained application (vichara), decision (adhimokkha), energy (viriya), zest (priti) and desire (chanda). Fourteen unwholesome universal mental factors exclusively appear in unwholesome thoughts. There are 10 more 'unwholesome occasional' mental factors that appear in certain thoughts, depending on whether the thought is related to greed, hatred and delusion. There are 25 mental factors which exclusively appear in wholesome thoughts. Within this category, abstinences, the illimitable and non-delusion are more related to higher-order thoughts pertaining to understanding of the ultimate truth. 
Table 1: Fifty-two mental factors.

\begin{tabular}{lll}
\hline Ethically variables (13) & Unwholesome (14) & Wholesome (25) \\
\hline Universals (7) & Unwholesome universal (4) & Universal beautiful (19) \\
Contact & Delusion & Confidence \\
Feeling & Shamelessness & Mindfulness \\
Perception & Fearlessness of wrong & Shame \\
Volition & Restlessness & Dread \\
One-pointedness & & Non-greed \\
Psychic life & Unwholesome occasional (10) & Non-hatred \\
Attention & Greed & Equanimity \\
& Wrong view & Tranquility of mental states \\
Occasional (6) & Conceit & Tranquility of mind \\
Initial application & Hatred & Lightness of mental states \\
Sustained application & Jealousy & Lightness of mind \\
Decision & Avarice & Pliancy of mental states \\
Energy & Worry & Pliancy of mind \\
Zest & Sloth & Adaptability of mental states \\
Desire & Torpor & Adaptability of mind \\
& Doubt & Proficiency of metal states \\
& & Proficiency of mind \\
& & Rectitude of mental states \\
& & Rectitude of mind \\
& & Abstinences (3) \\
& & Right speech \\
& & Right actions \\
& & Right livelihood \\
& & Illimitable (2) \\
& & Compassion \\
& & Appreciative joy \\
& & Non-delusion (1) \\
& Wisdom faculty \\
\hline & & \\
& & \\
& & \\
& &
\end{tabular}

Any thought that arises in the mind can be characterized by a combination of these mental factors. However, some combinations of mental factors do not form thoughts. For example, attachment and hatred do not co-exist in a thought.

\subsection{Thoughts}

As stated earlier, thoughts are formed by mental factors, and flow of thoughts manifest the mind. However, the story about thoughts goes beyond these fundamental definitions of mental factors and thoughts. More specifically, Abhidhamma is not limited to describe the relationship between 
thoughts and mental factors. Thoughts are also described in relation to feelings, roots, function, doors, objects and bases [33]. Each thought manifests the feeling of pleasure (sukha), pain (dukkha), joy (somanassa), displeasure (domanassa) or equanimity (upekkha). Each thought is also rooted in greed, hatred, delusion, non-greed, non-hatred or non-delusion. Thoughts have one or more functions (14), namely, rebirth-linking, life continuum, adverting, seeing, hearing, smelling, tasting, touching, receiving, investigating, determining, 'javana', registration and death. There are also doors, namely, eye door, ear door, tongue door, nose door, body door and mind door, through which each thought is created. Any thought is also associated with an object, namely, visible object, sound object, smell object, taste object, tangible object and mental object. The base is the physical support for the occurrence of thoughts. Note that base is different from doors, which are the channels for formation of thoughts. Abhidhamma also defines many other concepts including thought processes, description of matter, and relationship between mind and matter.

\section{COMPUTATIONAL ONTOLOGY OF MIND}

This section describes the design and implementation of ontology of mind from Buddhist philosophical system. Primarily, we have used Protégé toolkit [21] for design and development of the ontology. We have named our ontology as OntoBM, an acronym for Ontology of Buddhist theory of Mind.

\subsection{Design of OntoBM}

OntoBM has been designed with the principle that the mind can be modeled effectively through 52 mental factors as the basis for the whole modeling process. Thus, thoughts can be formed as permitted combinations of mental factors. At the top level, three major categories of mental factors, namely, ethical variable, unwholesome and wholesome have been modeled in OntoBM. The details of thoughts as feelings, roots, function, doors, objects and bases have also been annotated with each thought. OntoBM has been designed to be able to annotate the ontology at any time with further details of any node in the ontology. Furthermore, OntoBM is designed to connect with ontology query engine or applications such as multi-agent systems. Ontology Web Language (OWL) was used to write OntoBM into Protégé. Figure 1 shows a portion of the ontology of mental factors within Protégé environment.

\subsubsection{Reasoning on OntoBM}

Ontology being an inherently complex structure, reasoning on OntoBM is also a challenging task. To develop a reasoner on OntoBM, we used OWL API [35]. Within the context of OWL API, there are many OWL reasoners including Chainsaw [24], FaCT++ [25] and Pellet [26]. We have chosen Pellet reasoner because it is efficient in reasoning and querying. Also, it is freely available for downloading and readily compatible with Protégé environment.

\subsubsection{OntoBM interface design}

Graphical user interface of OntoBM has been designed to answer queries under different experimental conditions. The current interface of OntoBM primarily provides two views as Mental Factor View (MFV) and Thought View (TV). MFV allows to study or research by examining through mental factors. This view also allows the examining effect of modulation of mental factors on thoughts. In contrast, TV allows interaction with OntoMB through various thoughts as the inputs. Figure 2 depicts the MFV on OntoBM. 


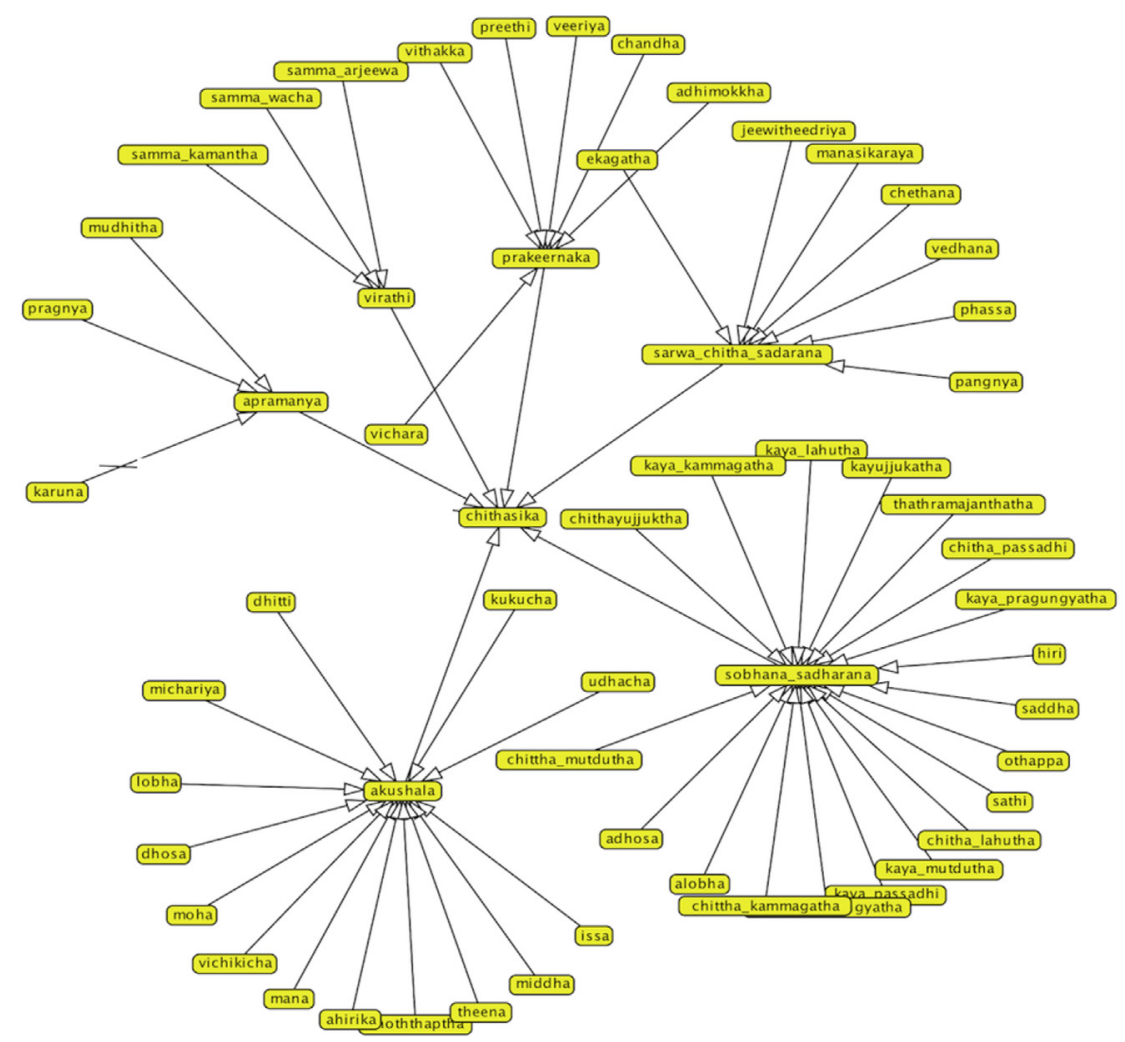

Figure 1: Ontology of mental factors.

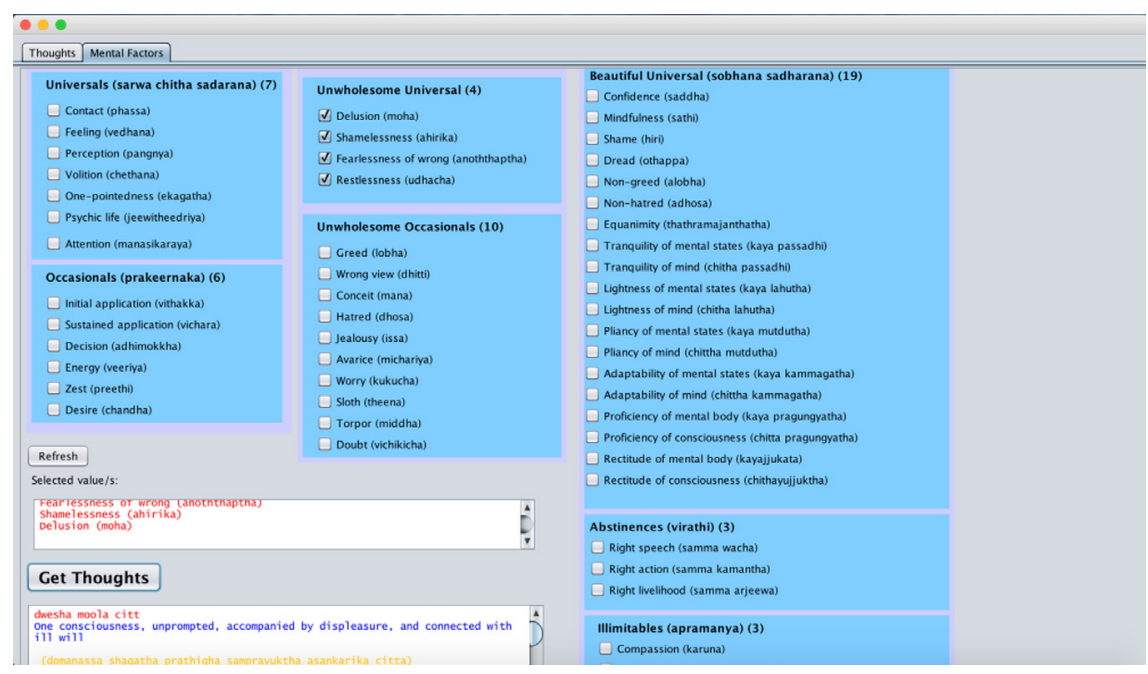

Figure 2: Mental factor view. 


\section{USING OntoBM}

OntoBM primarily allows learners and researchers to choose arbitrary combinations of mental factors to investigate whether such combinations could form a set of valid thoughts. When a user attempts to choose illegal (undefined) combinations of mental factors, for instance, greed with hatred, OntoBM does not allow continuing. On the other hand, when selecting a mental factor, OntoBM automatically sets the other mental factors that co-exist with the already selected mental factors. Thus, the OntoBM interface has some smart features, so that a user with zero knowledge in Buddhist theory of mind can still use the system. It should also be noted that even a person who knows Abhidhamma could not remember all associations of thoughts and mental factors off hand. Thus, OntoBM will be a learning tool as well.

Obviously, a knowledgeable researcher can introduce experimental combinations of mental factors and examine how the test case effects the formation of thoughts. If the selected combinations of mental factors cannot form any thoughts, the reasons will also be displayed by OntoBM. A researcher may also examine the mental factor which occurs in the largest number of unwholesome thoughts and which wholesome mental factor can counter it. In addition, a user can also select a thought and find its details such as mental factors, functions and bases. Further annotations to the OntoBM are still in progress. Depending on the researcher's creativity, many experiments can be done with OntoBM.

\section{CONCLUSION}

This paper describes a computational modeling of thought according to the Abhidhamma system in Buddhist psychology. We presented our research on the development of a computer model of mind from a Buddhist philosophical perspective of mind. This research has exploited Abhidhamma of Theravada Buddhist theory of mind to model the mind in terms of 52 mental factors, 89 thoughts and other related features of thoughts. Based on Abhidhamma, we have developed a computer model of ontology of mind, named as OntoBM. We have illustrated with MFV and TV, which can be used to research into behavior of mental factors and thoughts. OntoBM can be primarily used to examine combinations of mental factors that can form thoughts, associations among mental factors and details of thoughts. More importantly, researchers and scholars can also use OntoBM to experiment with the effect of modulation of mental factors on the formation of thoughts. As such, OntoBM can be used by many individuals including learners of Buddhist model of mind, researchers in brainmind studies, meditation practitioners/teachers/researchers and psychologists. While in the development process, the results of experiments on OntoBM tested against the Abhidhamma text is encouraging. As further work, we are working on the development of a versatile mind simulator based on the ontology of mind, OntoBM, presented in this paper.

\section{REFERENCES}

[1] Rzevski, G. \& P. Skobelev, Managing Complexity, WIT Press, 2014.

[2] Dworschak, M, Neurotechnology: Growing a Brain in Switzerland, Spiegel Online, 16 Feb. 2007.

[3] B.C.Q.C., Live Science Contributor, Uploading the Mind: Could a Digital Brain Feel Pain? LiveScience.com. [online]. Available: http://www.livescience.com/46052-mind-uploadingethics.html [accessed: 06-Nov-2014].

[4] Markram, H, The Blue Brain Project, Nat. Rev. Neurosci., 7(2), pp. 153-160, Feb. 2006.

[5] Brainsimulator,Softonic.[Online].Available:http://en.softonic.com/s/brain-simulator[accessed: 09-Nov-2014]. 
[6] Cao, M., Wang, J.-H., Dai, Z.-J., Cao, X.-Y., Jiang, L.-L, Fan, F.-M., Song, X.-W., Xia, M.-R., Shu, N., Dong, Q., Milham, M.P., Castellanos, F.X., Zuo, X.-N, \& He, Y. Topological organization of the human brain functional connectome across the lifespan. Dev. Cogn. Neurosci., 7, pp. 76-93, Jan. 2014.

[7] Campbell, I.G., EEG Recording and Analysis for Sleep Research. Curr. Protoc. Neurosci. Editor. Board Jacqueline N Crawley Al, Chapter 10, Unit10.2, Oct. 2009.

[8] Priori, A., Brain polarization in humans: a reappraisal of an old tool for prolonged non-invasive modulation of brain excitability. Clin. Neurophysiol. Off. J. Int. Fed. Clin. Neurophysiol., 114(4), pp. 589-95, 2003. doi: http://dx.doi.org/10.1016/S1388-2457(02)00437-6

[9] Steward, H., The Ontology of Mind: Events, Processes, and States. Clarendon Press Oxford Philosophical Monographs, 1997.

[10] Stoljar, D., \& Steward, H., The Ontology of Mind: Events, States and Processes. Philos. Rev., 108(3), p. 418, July 1999.

[11] Karunananda, A.S., Using an eastern philosophy for providing a theoretical basis for some Heuristics used in artificial neural networks. Malays. J. Comput. Sci., 15(2), 2002.

[12] Karunananda, A., Computer Modelling of thought processes. Int. J. Comput. Appl. Technol., 6(2-3), pp. 135-140, 1993.

[13] Narada, M.T., A Manual of Abhidhamma. Buddhist Missionary Society, 1979.

[14] Prigogine, I., The End of Certainty, 1 edition. New York: Free Press, 1997.

[15] Prigogine, I., Is Future Given? World Scientific, 2003.

[16] Herculano-Houzel, S., The remarkable, yet not extraordinary, human brain as a scaled-up primate brain and its associated cost. Proc. Natl. Acad. Sci., 109(1), pp. 10661-10668, June 2012.

[17] Dave Evans, The Internet of Things How the Next Evolution of the Internet Is Changing Everything. 2011.

[18] Gruber, T.A., A Translation Approach to portable Ontology Specification. Knowl. Acquis., 5(2), pp. 199-220, 1993. doi: http://dx.doi.org/10.1006/knac.1993.1008

[19] Luciano, J.S., Andersson, B., Batchelor, C., Bodenreider, O., Clark, T., Denney, C.K., Domarew, C., Gambet, T., Harland, L., Jentzsch, A., Kashyap, V., Kos, P., Kozlovsky, J., Lebo, T., Marshall, S.M., McCusker, J.P., McGuinness, D.L., Ogbuji, C., Pichler, E., Powers, R.L., Prud'hommeaux, E., Samwald, M., Schriml, L., Tonellato, P.J., Whetzel, P.L., Zhao, J., Stephens, S. \& Dumontier, M., The Translational Medicine Ontology and Knowledge Base: driving personalized medicine by bridging the gap between bench and bedside, J. Biomed. Semant, 2(2), p. S1, May 2011.

[20] Rodriguez-Rocha, B.D., Castillo-Barrera, F.E. \& Lopez-Padilla, H., Knowledge Capitalization in the Automotive Industry Using an Ontology Based on the ISO/TS 16949 Standard, in Electronics, Robotics and Automotive Mechanics Conference, Los Alamitos, CA, USA, 2009, pp. 100-106.

[21] Protégé. http://protege.stanford.edu, 2012.

[22] Noy, N.F. \& Musen, M., PROMPT: Algorithm and tool for automated ontology merging and alignment, Proc. 17th Natl. Conf. Artif. Intell. AAAI-2000 Austin TX, 2000.

[23] Acampora, G., Avella, P., Loia, V., Salerno, S. \& Vitiello, A., Improving ontology alignment through memetic algorithms, in 2011 IEEE International Conference on Fuzzy Systems (FUZZ), pp. 1783-1790, 2011. doi: http://dx.doi.org/10.1109/FUZZY.2011.6007517

[24] Tsarkov, D. \& Palmisano, I., Chainsaw: A Metareasoner for Large Ontologies. in CEUR Workshop Proceedings, 2012.

[25] Abburu, S., A Survey on Ontology Reasoners and Comparison. Int. J. Comput. Appl., 57(17), 2012. 
[26] Sirin, E., Parsia, B., Grau, B.C., Kalyanpur, A. \& Katz, Y., Pellet: A practical OWL-DL reasoned. Web Semant. Sci. Serv. Agents World Wide Web, 5(2), pp. 51-53, June 2007.

[27] Wooldridge, M., An Introduction to MultiAgent Systems. John Wiley \& Sons, 2009.

[28] Rzevski, G., Modelling Large Complex Systems Using Multi-agent Technology, pp. 434-437, 2012. doi: http://dx.doi.org/10.1109/snpd.2012.138

[29] Hongqiao, Y., Xihua, L., Fei, W. \& Weizi, L. Multi-agent based modeling and simulation of complex system in hospital, in 16th International Conference on Industrial Engineering and Engineering Management, 2009. IE EM '09, pp. 1759-1763, 2009.

[30] Belgacem, S. \& Fates, N., Robustness of Multi-agent Models: The Example of Collaboration between Turmites with Synchronous and Asynchronous Updating, Complex Syst., 21, 2012.

[31] Singh, A., Juneja, D. \& Sharma, A.K., Agent Development Toolkits, Int. J. Adv. Technol., 2(1), pp. 158-164, 2011.

[32] Nyanaponika, T., Ahidhamma Studies - Research in Buddhist Psychology. Wisdom Publications, 2013.

[33] Bodhi, B., Abhidhammatha Sangaha: a comprehensive mannula of Abhidhamma. Onalaska: BPS Pariyatti Edition, 2000.

[34] Kalupahana, D.J., The principles of Buddhist Psychology. State University of New York Press, 1987.

[35] Horridge, M. \& Bechhofer, S., The owl api: A java api for owl ontologies, Semantic Web, 2(1), pp. 11-21, 2011. 